\title{
Conditioned Medium From M1 Macrophages Induces the Migration of Neural Stem Cells Through MCP-1/CCR2 and ERK Pathway
}

\section{Zhijian Cheng ( $\square$ czj0606@126.com )}

Xi'an Jiaotong University https://orcid.org/0000-0003-4769-6335

\section{Wen Zhu}

The first people's hospital of xianyang city

\section{Xijing He}

Xi'an Jiaotong University

Cao Kai

Xi'an Jiaotong University

Jin Li

Xi'an Jiaotong University

Haopeng Li

Xi'an Jiaotong University

Guoyu Wang

Xi'an Jiaotong University

\section{Research Article}

Keywords: neural stem cells, bone marrow derived macrophage, conditioned medium, migration, transplantation

Posted Date: December 3rd, 2021

DOI: https://doi.org/10.21203/rs.3.rs-1116302/v1

License: (a) (i) This work is licensed under a Creative Commons Attribution 4.0 International License. Read Full License 


\section{Abstract}

After spinal cord injury, grafted neural stem cells (NSCs) can migrate toward injured area, where there are a large number of bone marrow derived macrophage. However, little is known about the effect of M1 macrophage from bone marrow on NSCs migration and the mechanism responsible for migratory responses of grafted NSCs in vitro and in vivo. Migration of NSCs were conducted by using the transwell chamber and SCl model. Conditioned medium from M1 macrophages (M1-CM) can attract NSCs migration in vitro. The number of migrated C-C chemokine receptor 2 (CCR2) -/- NSCs induced by M1-CM were significantly decreased compared with wild type (WT) NSCs $(P<0.05)$. Furthermore, compared with M0 macrophages, the production of MCP-1 by M1 macrophages was significantly increased and M1-CM treated with anti-MCP-1 antibody to neutralize MCP-1 can lead to a significant reduction of NSCs migration $(P<0.05)$. In addition, Western blot showed that ERK1/2 was dramatically activated following the stimuli of M1-CM and then ERK inhibitor can also inhibit M1-CM-mediated MSCs migration. Finally, CCR2 was needed for grafted NSCs migration toward injured area. Bone marrow derived M1 macrophage persisted within the epicenter of injured area and the expression of MCP-1 mRNA was significantly increased 7days after SCI $(P<0.05)$. These results demonstrated the effect of $\mathrm{M} 1$ macrophages on NSCs migration and the important role of MCP-1/CCR2 and ERK signal pathway on M1-CM-induced NSCs migration.

\section{Introduction}

Neural stem cells (NSCs) are defined by their ability to self-renew and to generate the main phenotypes of central nervous system (CNS) cells, which are neurons, astrocytes, and oligodendrocytes[1, 2].

Transplantation of NS/PCs is promising treatment for spinal cord injury (SCI)[3-5]. NSCs migration is an essential process for the development of the central nervous system (CNS). Recently, it's has also been showed that transplanted NSCs migrate to sites of pathological area such as various types of CNS diseases (i.e., spinal cord injury and ischemia) [6-8]. The migration capability is very important for endogenous or exogenous NSCs to repair the damaged tissue $[9,10]$. Identification of NSCs migration would not only aid a better understanding of the mechanisms responsible for injury-mediated NSCs migration but also have clinical implications for stem cell-based therapeutic strategies for spinal cord injury (SCl).

Spinal cord injury (SCl) is a traumatic event that involves not just an acute physical injury, but also inflammation driven secondary injury[11]. Macrophages play a very important role in secondary injury. After $\mathrm{SCl}$, blood monocytes are recruited by primary chemokines and cytokines to injured area 2-3days post-injury and bone-marrow derived macrophages accumulate in the injury site about 1 week after $\mathrm{SCI}[12]$. Regardless of the cause of $\mathrm{SCl}$, there are a lot of macrophages persisting at injury site indefinitely. Kigerl et al reported that the rapid response and maintenance of $\mathrm{M} 1$ and the transient existence of M2 at the injured spinal cord area leaded to the second damage and regulate the differentiation of NSCs[13]. M1 macrophages could regulate the differentiation and proliferation of neural stem/progenitor cells, and may be relative to migration of NSCs. 
Although little is known of the mechanisms responsible for the injury-mediated NSPC migration, there are a variety of cytokines and growth factors, such as monocyte chemoattractant protein (MCP-1), CXC chemokine ligand (CXCL)12, stromal cell-derived factor-1 (SDF-1), fibroblast growth factor (FGF), and epidermal growth factor (EGF), have been shown to influence NSCs migration[14-16]. Chemokine C-C motif receptor 2 (CCR2) is a G protein-coupled receptor. Previous studies showed NSCs express CCR2 and play a crucial role on NSCs migration. MCP-1 activates the migration capacity of NSCs in vitro[17].

To date, little is known about the role of bone marrow derived M1 macrophages on the migration of NSCs after transplanted into injured spinal cord, and the precise molecular mechanisms involved in this process remain unclear. We hypothesized that M1 macrophage promotes migration of NSCs though MCP-1/CCR2 in vitro as well as in vivo. This study analyzed (1) the effect of M1-CM on the migration of NSCs and (2) the role of MCP-1, CCR2 and ERK pathway on M1-CM-induced NSCs migration in vitro and in vivo.

\section{Materials And Methods}

\section{Animals}

CCR2-/- mice (B6.129S4-Ccr2tm1 Ifc) and wild type C57BL/6 mice were purchased from Jackson Laboratory (Bar harbor, ME) and mice were housed in pathogen-free Animal Experiment Center of Xi'an Jiaotong University's college of medicine. All experimental procedures were approved by Xi'an Jiaotong University's Animals Care and Use Committee (ACUC) and performed in accordance with the guide for the care and use of laboratory animals.

\section{Reagents and Antibodies}

Dulbecco's Modified Eagle's Medium, DMEM/F12 (1:1) and B-27supplement were purchased from Invitrogen (Carlsbad, CA, USA). Basic fibroblast growth factor (bFGF), interferon-y (IFN- - ), epithelial growth factor (EGF) and anti-TUJ1 antibody were obtained from Millipore (Billerica, MA, USA). Hybridoma cell lines of Mac-2 and F4/80 were obtained from the American Tissue Culture Collection (ATCC, Manassas, VA, USA). Anti-MCP-1 antibody, anti-nestin antibody and anti-glial fibrillary acidic protein (GFAP) antibody were purchased from Abcam (Cambridge, MA, USA), and anti-iNOS antibody from BD

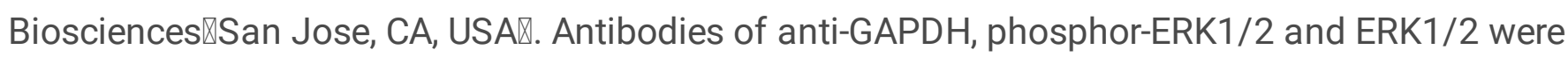
purchased from Cell Signaling Technology (Danvers, MA, USA). All secondary antibodies were purchased from Invitrogen (Carlsbad, CA, USA).

\section{Preparation of Mouse Bone Marrow-derived Macrophage}

Mouse BMDMs were prepared from C57BL/ 6 wild type mice as previously described[18]. Briefly, BMDMS were harvested from mice 6-8 weeks of age with 7days culture in DMEM supplemented with $5 \%$ newborn calf serum (NCS) (Rocky Mountain Biologicals), 15\% conditioned medium from L929 cells (a source of macrophage colony-stimulating factor), and 1\% penicillin/streptomyclin-solution (Corning, USA). 


\section{Induction of M1 Macrophage and Conditioned medium preparation}

To promote polarization into M1 macrophages, BMDMs were treated with $10 \mathrm{ng} / \mathrm{ml}$ interferon- $\mathrm{Y}$ (IFN$\gamma$, Millipore, Billerica, MA, USA) for 24h[19, 20]. M0 macrophages weren't treated with IFN- $\gamma$ except for a change in the macrophage medium. After 24h, macrophages were washed three times with phosphatebuffered saline, changed with fresh medium including 2\% B-27 supplement (Gibco) and then cultured for 24h. Conditioned medium (CM) from M0 macrophages and M1 macrophages were prepared by centrifuging collected medium at 2500RPM for $19 \mathrm{~min}$ and filtering through a $0.4-\mu \mathrm{m}$ filter (Corning). While, culture medium (without BMDMs) was used as control medium in this study.

\section{NSCs Isolation and Culture}

Primary NSCs were isolated and cultured from fetal wild type C57BL/6 mice and CCR2-/- mice (B6.129S4-Ccr2 ${ }^{\text {tm1 } 1 \text { ff }}$ ) cortices at embryonic day 14, as previously described[21]. In brief, cerebral cortex was collected and mechanically dissociated into single cell. Then cells were seeded in DMEM/F12 supplemented wiht $20 \mathrm{ng} / \mathrm{ml}$ EGF, $10 \mathrm{ng} / \mathrm{ml} \mathrm{bFGF,} \%$ B-27 supplement and 1\% penicillin/streptomycin, and incubated at $37^{\circ} \mathrm{C}$ on a humidified atmosphere containing $5 \% \mathrm{CO} 2$.

\section{Transwell Migration Assay of NSCs}

Migration of NSCs were conducted by using the transwell chamber with a pore size of $8 \mu \mathrm{m}$ (Neuro probe) as described previously. After 8 - $\mu \mathrm{m}$ pore membrane coated with $0.01 \%$ poly-l-lysine, $20 \mu \mathrm{l}$ of $5 \times 10^{6} / \mathrm{ml}$ NSCs were added to upper wells of the transwell chamber. DMEM with $2 \%$ B-27 supplement (control medium), conditioned medium from M0 macrophages(M0-CM), conditioned medium from M1 macrophages (M1-CM) and other reagents dissolved in DMEM with 2\% B-27 supplement were located at the lower wells of the chamber. After $12 \mathrm{~h}$, the number of cells, that migrated through the filters were fixed and subjected to Giemsa staining, were analyzed by counting three independent field of each condition.

\section{MTT Assay}

MTT (3-(4, 5-Dimethylthiazol-2-yl)-2,5-diphenyl-tetrazolium bromide) assay was performed to identify whether M1-CM influences the proliferation of NSCs. After treated with M1-CM, M0-CM or control medium for $12 \mathrm{~h}$, cells were incubated with MMT solution ( $5 \mathrm{mg} / \mathrm{ml}$, Sigma-Aldrich, St.Louis, MO, USA) for $5 \mathrm{~h}$, and then added $200 \mu \mathrm{l}$ of DMSO to dissolve crystals. The absorbance was measured at $550 \mathrm{~nm}$ using a microplate reader (Bio-Rad, Hercules, CA, USA) and relative proliferation rates were observed by comparing the optical density value of experimental samples to that of control sample.

\section{Enzyme-linked immunosorbent assay (ELISA)}

Quantitative measurement of MCP-1 was analyzed and determined using MCP1 ELISA kit (Abcam, Cambridge, MA, USA) following the manufacturer's instruction. The absorbance was measured at $450 \mathrm{~nm}$ and MCP-1 concentration was observed by comparing absorbance to standard curves. 


\section{Western Blot}

After treated with M1-CM, NSCs were washed with ice-cold PBS and lysed in RIPA buffer containing a phosphatase inhibitor and proteinase inhibitor cocktail (Amresco, Solon, OH, USA). Western blot assay was carried out following standard procedure. Briefly, total NSCs proteins were adjusted to equal protein concentrations, loaded onto SDS- polyacrylamide gel electrophoresis (SDS-PAGE) and then transferred to PVDF membranes. After blocking in 5\% milk in Tris-buffered saline containing $0.1 \%$ Tween 20 (TBST) for

$1 \mathrm{~h}$ at RT, membranes were incubated with primary antibodies solution overnight at $4^{\circ} \mathrm{C}$. Membranes were then placed in appropriate secondary antibody for $1 \mathrm{~h}$ at room temperature after rinsing in TBST. Blots were visualized with the ECL plus western blot detection system (GE Healthcare, UK).

\section{Phalloidin staining}

The distribution and expression of F-actin was performed using CF488-conjugated phalloidin (89427-132, Biotium, Fremont, CA, USA) according to the manufacturer's protocol. Briefly, NSCs were treated with control medium and M1-CM, then washed three times with PBS and fixed with $4 \%$ paraformaldehyde (PFA) in PBS for 15 min at room temperature. Following by permeabilization with $0.5 \%$ Triton $\mathrm{X}-100$ in PBS for $10 \mathrm{~min}$ at room temperature, cells was washed and incubated with phalloidin and DAPI at room temperature for $20 \mathrm{~min}$. Finally, the coverslips were washed, mounted and viewed under EVOS fluorescent microscope (Life technologies, Carisbad, CA USA).

\section{SCI Model and NSCs Transplantation}

Contusion SCI was induced using a NYU impactor[22]. Adult female C57BL/ 6 mice $(20 \pm 2.5 \mathrm{~g}$ in body weight) were anesthetized with $100 \mathrm{mg} / \mathrm{kg}$ ketamine and $10 \mathrm{mg} / \mathrm{kg}$ xylazine through intraperitoneal injection. The tenth thoracic (T10) spinal cord segment was exposed and a contusion injury of the T10 vertebral cord was induced by dropping a $5-\mathrm{g}$ rod $6.25 \mathrm{~mm}$ onto the spinal cord. Postoperative care such as manual bladder emptying was performed as previously described.

Then, NSCs were transplanted into mice with $\mathrm{SCl}$ to examine their migration in vivo. In brief, CCR2-/NSCs and WT NSCs were pre-labeled with 5(6)-carboxyfluorescein diacetate N-succinimidyl ester (CFSE, Sigma-Aldrich, St.Louis, MO, USA) according to manufacture's protocol before cells transplantation. 7 days after $\mathrm{SCl}$, a total volume of $0.5 \mu \mathrm{l}$ of cell suspension containing $5 \times 10^{4} \mathrm{NSCs}$ was microinjected at either side of the spinal cord contusion $2 \mathrm{~mm}$ away using a Hamilton syringe.

\section{Real Time PCR}

Mice were sacrificed and spinal cords were immediately extracted 7days after $\mathrm{SCl}(\mathrm{n}=5$ each group). Total RNA was isolated from the indicated tissues via TRI reagent (Sigma-Aldrich, St.Louis, MO, USA) and then reverse-transcribed into cDNA using Fist strand cDNA Synthesis kits (Roche, Basel, Switzerland). The following primer sets were as following: MCP-1 (5'-ATGCAGGTCCCTGTCATGCTT-3' and 5'CATTGGGATCATCTTGCTGGT-3') and GAPDH (5'- ATCAACGACCCCTTCATTGACC-3' and 5'- 
CCAGTAGACTCCACGACATACTCAGC-3'). We assayed the expression of target gene mRNA by using the ABI 7900HT Fast real-time PCR detection system (Applied Biosystems, UK) and expression was normalized to GAPDH for each sample.

\section{Immunohistochemistry}

The spinal cord was fixed with $4 \%$ paraformaldehyde in phosphate-buffered saline (PBS) overnight at $4^{\circ} \mathrm{C}$, incubated with $30 \%$ sucrose solution at $4^{\circ} \mathrm{C}$ until tissue sunk, and then frozen in OCT compound. 8um-thick parasagittal sections were prepared by using a cryostat microtome and attached to poly-llysine-coated glass slides. For immunofluorescence staining, samples were blocked with $1 \%$ BSA in PBS containing $0.3 \%$ Triton $\mathrm{X}-100$ at room temperature for $1 \mathrm{~h}$, and incubated with primary antibodies at $4^{\circ} \mathrm{C}$ for $12 \mathrm{~h}$. After washed with PBS, the sections were incubated with secondary antibodies at room temperature for $1 \mathrm{~h}$. All the procedures for negative controls were performed in the same manner, just except incubation of primary antibodies._EVOS fluorescent microscope (Life technologies, Carisbad, CA USA) was used to observe and capture the images.

\section{Statistical Analysis}

The results were reported as mean \pm standard deviation (SD). All statistical data was analyzed using SPSS 22.0 software program (StatSoft, Tulsa, OK). The statistical significance was evaluated using Student's unpaired t-test and Statistical significance was assumed if $P<0.05$.

\section{Results}

\section{Culture and characterization of NSCs}

After 7days cultured in serum free media including B27, EGF and bFGF, NSCs proliferated and formed neurosphere (Figure 1A). For identification of NSCs, the neurosphere after two passages were stained with nestin[23], a marker of NSCs, and DAPI was used to mark nuclei. The result showed that the neurosphere was nestin positive (Figure 1B). To further examined the capacity of generating the main phenotypes of CNS cells including neurons and astrocytes, NSCs were seeded on the PLL-coated coverslips and cultured for 5days in differentiation medium which were DMEM/F12 medium supplementing with 1\% FBS and 2\% B27, and then stained with GFAP for astrocyte and Tuj 1 for neuron. The result showed that the cells expressed TUJ1 (Figure 1C) and GFAP (Figure 1D), which indicated that the multipotency of these NSCs.

\section{Conditioned medium from M1 macrophages (M1-CM) induced NSCs migration}

To determine whether M1-CM can induce the migration of NSCs, we induced M1 macrophages by IFNY (10ng/ml, Millipore) and performed a transwell migration assay in vitro. Compared with control medium, a significant increase of migration of NSCs was observed in the presence of M1-CM and M0-CM $(P<0.05$, Figure 2A and 2B). Meanwhile, the number of migrated NSCs toward M1-CM was significantly higher than that of migrated NSCs toward M0-CM ( $P<0.05$, Figure 2A and 2B). MTT assay was performed 
to exclude possible influence from the proliferation of NSCs. MTT result showed M1-CM and M0-CM had no significant effect on proliferation of NSCs within $12 \mathrm{~h}$ ( $P>0.05$, Figure $2 \mathrm{C})$. Therefore, these results indicated that M1-CM could attract NSCs migration in vitro.

\section{M1-CM promoted NSCs migration via CCR2}

CCR2 is very important for the migration of NSCs[24]. In order to investigate whether CCR2 is involved in the regulation of M1-CM-induced NSCs migration, the effect of M1-CM on the migration of NSCs isolated from CCR2-/- mouse used in migration assay was tested. At the beginning, expression of CCR2 in WT NSCs and CCR2-/- NSCs was confirmed by reverse transcription polymerase chain reaction (Figure 3A). As showed in Figure 3B and 3C, the migration assay results demonstrated that the chemotactic effect of M1-CM on the migration of NSCs was significantly inhibited in the CCR2-/- NSCs group compared with WT NSCs $(P<0.05)$.

\section{M1-CM induced NSCs migration by secretion of MCP1}

CCR2 is known as the only high-affinity receptor for MCP-1[25]. Widera et al also found that MCP-1 can act as a chemotactic factor for adult NSCs[26]. In order to investigate whether M1 macrophages induce NSCs migration by secreting MCP-1, we examined the production of MCP-1 by M1 macrophages using enzyme-linked immunosorbent assay (ELISA). The ELISA results showed that BMDMs stimulated by IFN$\gamma$, dramatically increased the production of MCP-1 compared to BMDMs which were cultured without treatment of INF- IFN-y ( $P<0.05$, Figure 4A). Next, M1-CM was treated with anti-MCP-1 antibody to neutralize the MCP-1. A significant reduction of migration observed in M1-CM+ anti-MCP-1 antibody group in comparison to M1-CM group ( $P<0.05$, Figure 4B and $4 \mathrm{C}$ ). Taken together, these results suggested M1 macrophages probably secreted MCP-1, Which activated the CCR2 receptor, to attract the migration of NSCs in vitro.

\section{Migration of NSCs toward M1-CM was dependent on ERK pathway}

ERK pathway has been implicated in the migration of numerous cell types including neural stem/progenitor cells and mesenchymal stem cells. To determine the effect of ERK pathway on the migration of NSCs toward M1-CM, PD98059, an inhibitor of ERK pathway[27], were used in this study and found that it could inhibit the migration of NSCs toward M1-CM $(P<0.05$, Figure 5A and 5B). Furthermore, the treatment of NSCs with M1-CM could stimulate phosphor-ERK1/2 and the M1-CM-induced expression of P-ERK1/2 in NSCs was markedly decreased after treated with PD98059 (Figure 5C). Collectively, these results convinced us thatM1-CM induced NSCs migration by activation of ERK1/2.

\section{F-actin participated in the migration of NSCs}

F-actin is important for shaping cell morphology and regulating cell migration[28]. Next, to determined whether F-action was involved in NSCs migration induced by M1-CM, fluorescent phalloidin staining were used to investigate the distribution of F-actin in NSCs. Phalloidin is a bicyclic peptide that binds to specifically to the F-actin and doesn't bind to G-actin[29]. F-actin was expressed throughout the 
cytoplasm. While, compared with the control group and M1-CM group, F-actin structures were distributed around the periphery of the cells and the expression of F-actin were significantly increased(Figure 6).

\section{Migration of local transplanted NSCs was dependent on CCR2 after SCI}

Furthermore, in order to assess the effect of CCR2 and BMDMs on the migration of grafted NSCs after injury, the microinjection of NSCs besides the injury site was conducted 7days after SCl. As reported, bone marrow derived macrophages (BMDMs) could migrate to the injured area about 3 days after the injury and accumulate within the epicenter of the injured site about 7days after SCI[12], so migration of NSCs in vivo was performed at this time point. The results showed that transplanted WT NSCs migrated from injection site into the injured area 5days post transplantation, while the movement of CCR2-/- NSCs was lower than WT NSCs 5days after cell transplantation (Figure 7B). As showed in Figure 7A1 and 7B1, WT NSCs were found in the injury site identified by a lot of $F 4 / 80$ (a microglia/macrophages marker) positive signal (Red), but no GFP signal were observed within injury area in CCR2-/- NSCs group.

\section{Expression of M1 macrophage and MCP-1 mRNA 7days after SCI}

Conditioned medium from M1 macrophages, which was induced by IFN- $\gamma$, attracted the migration of NSC in vitro possible by secreting MCP-1, so we detected the expression of M1 macrophage marker and mRNA level of MCP-1 7days after SCl. To test the phenotype of macrophage 7days after SCl, M1 macrophages were identified by double Mac-2 (a marker of macrophage) and inducible nitric oxide synthase (iNOS, a marker of M1 macrophages) staining. The result showed that most of macrophages were Mac2+/iNOS+ cells 7 days after injury (Figure 8A). Next, we evaluated the expression of MCP-1 mRNA in injured tissue and found that mRNA level of MCP-1 was significantly increased in the SCI group than that of sham group at the same time $(P<0.05$, Figure $8 \mathrm{~B})$. Taken together, $\mathrm{M} 1$ macrophages may not only induce NSCs migration in vitro, but also play an important role on the grafted NSCs migration.

\section{Discussion}

Many in vivo studies have shown that NSCs, which are able to migrate toward injury sites of the CNS, may act as a delivery tool for tissue repair and regeneration $[8,30]$. In this study, we investigated the effects on conditioned medium from M1 macrophage (M1-CM) on migration of NSCs and the possible signal molecules in this migratory process.

Macrophages are of great importance for inflammatory response after $\mathrm{SCl}$ and there are two main subsets of macrophages, classical macrophages M1 and alternative macrophages M2[31]. Classical activation involves the induction of M1 macrophages by toll-like receptor ligands or pro-inflammatory cytokines IFN- $\gamma$. These cells produce high levels of oxidative metabolites (e.g., superoxide and nitric oxide), inducible nitric oxide synthase (iNOS), chemokine (C-C motif) ligand 12 (CCL12), and proinflammatory molecules such as tumor necrosis factor-a (TNF- $\alpha$ ), interleukin-1 $\beta$ (IL-1 $\beta$ ), interleukin-6 (IL-6) and interleukin-12 (IL-12) that are essential for host defense and tumor cell killing but that also cause damage to healthy cells/tissue[32-34]. 
Results of the transwell migration assay showed that NSCs were seeded in the upper chamber, control medium or M-CM were placed in the lower chamber, and then the number of migrated NSCs toward M1$\mathrm{CM}$ was significantly increased compared with $\mathrm{MO}-\mathrm{CM}$ and control medium. The results confirmed that M1-CM had the capacity to induce NSCs migration in vitro. In vivo result showed that when BMDMs accumulated within the injury site 7days after injury and most of them were M1 macrophage which were identified by expressing both iNOS (a M1 macrophage marker) and Mac-2 (a macrophage marker), WT NSCs were local injected into spinal cord and found that they can migrate toward injury area. Therefore, we speculated that the injury-mediated NSCs migration toward damaged area may be partly due to the effect of M1 macrophages 7days after SCl. The migration of endogenous and exogenous NSCs may be affected by other factors in vivo. Previous studies have reported that hypoxia-induced astrocytes and activated microglia can induce NSCs migration in vitro[14,35]. Further studies are needed in order to get more evidences showing M1 macrophages induce NSCs migration in vivo.

Chemokines are major regulators of cell trafficking and adhesion. Numerous previous studies had shown that interaction between MCP-1 and its receptor CCR2. The binding of MCP-1 to CCR2 initiated divergent signaling pathways downstream of ligand binding, which had been already known to control varioustypes of normal and pathological cell migrations[36-38], also required for the migration of stem cells[39, 40]. In this study, to investigate the mechanism responsible for M1-CM-mediated NSCs migration, we compared the migration between WT NSCs and CCR2-/- NSCs in vitro and in vivo. The results showed that CCR2 played a very important role on the M1-CM-induced NSCs migration in vitro, as well as on the injury-mediated NSCs migration at sub-acute SCI. CCR2 is the only known high-affinity receptor for MCP-1. We found that BMDMs stimulated by IFN- $\gamma$ could increase the secretion of MCP-1 and lead to increasing migration of NSCs. Our results are in line with a previous report showing CCR2 expression in nestin-positive neurosphere cultures and MCP-1 can activate the migration capacity of NSCs[26]. Thus, we confirmed that M1-CM can induce NSCs migration by means of MCP-1/CCR2. It's known that chemokines are produced at sites of injury. Our RT-PCR result showed that the expression of MCP-1 mRNA was significantly increased at 1 week after SCl. Similarly, MCP-1 expression can be induced by mechanical injury within the brain[41, 42]. Therefore, MCP-1 and CCR2 were needed for grafted NSCs migrated from injection site to injury area after SCl.

Mitogen-activated protein kinase (MAPK) signaling pathway including ERK1/2, p38and JNK molecules have been reported to control major cell function[43, 44]. ERK1/2 molecule is an important molecule in the bridge between cell membrane and nucleus which could be activated via integrins, growth factors and so on, and plays an essential role in controlling gene transcription[45]. ERK1/2 control cell migration of human neural progenitor cells[46, 47]. In our study, we found that M1-CM strongly activated ERK1/2 phosphorylation. At the same time, PD98059 can abolish the strong phosphorylation of ERK1/2. Result from transwell assay confirmed our hypothesis, because PD98059 can significantly prevent M1-CMinduced migration of NSCs. Collectively, these results implied that ERK1/2 was an important signal molecule mediating M1-CM-induced NSCs migration in vitro. 


\section{Conclusion}

In summary, we demonstrated that M1-CM could promote NSCs migration in vitro. MCP-1/CCR2 and ERK1/2 signal pathway were involved in M1-CM-mediated NSCs migration. Furthermore, the migration of grafted NSCs toward injured area may be due to M1 macrophage within injured area depending on the MCP-1 and CCR2. Deep and detailed investigations on effects of BMDMs on the migration of NSCs will not only lead to a better understanding of the molecular mechanism of the interaction between NSCs and macrophages, but also provide an important theoretical guidance to develop NSCs as an efficient treatment for SCl.

\section{Declarations}

\section{Acknowledgments:}

Supported by National Natural Science Foundation of China Grand: No. 81801237, Shaanxi province natural science basic research program of China: N0.2018JQ8039, and the Fundamental Research Funds for the Central Universities of China: NO sxzy012019138.

\section{Data Availability Statement}

All data used during the study appear in the submitted article.

\section{Disclosure of conflict of interest:}

None

\section{Author Contributions:}

Zhijian Cheng and Xijing He conceived and designed the experiments; Zhijiian Cheng performed the biological experiments; Guoyu Wang and Haopeng Li prepared the reagents, materials and analysis tools; Zhijian Cheng, CaoKai, and Jin Li analyzed the data. Zhijian Cheng, Wen Zhu and Xijing He contributed to the writing of the paper.

\section{References}

1. Ourednik $\mathrm{J}$ et al (2002) Neural stem cells display an inherent mechanism for rescuing dysfunctional neurons. Nat Biotechnol 20(11):1103-1110

2. Okano H (2002) Stem cell biology of the central nervous system. J Neurosci Res 69(6):698-707

3. Hwang I et al (2016) Intrathecal Transplantation of Embryonic Stem Cell-Derived Spinal GABAergic Neural Precursor Cells Attenuates Neuropathic Pain in a Spinal Cord Injury Rat Model. Cell Transplant 25(3):593-607

4. Yokota K et al (2015) Engrafted Neural Stem/Progenitor Cells Promote Functional Recovery through Synapse Reorganization with Spared Host Neurons after Spinal Cord Injury. Stem Cell Reports 
5(2):264-277

5. Salewski RP et al (2015) Transplantation of neural stem cells clonally derived from embryonic stem cells promotes recovery after murine spinal cord injury. Stem Cells Dev 24(1):36-50

6. Kimura A et al (2007) Essential roles of sphingosine 1-phosphate/S1P1 receptor axis in the migration of neural stem cells toward a site of spinal cord injury. Stem Cells 25(1):115-124

7. Park DH et al (2009) Inflammation and stem cell migration to the injured brain in higher organisms. Stem Cells Dev 18(5):693-702

8. Dai J et al (2013) Migration of neural stem cells to ischemic brain regions in ischemic stroke in rats. Neurosci Lett 552:124-128

9. Theus MH et al (2010) EphB3 limits the expansion of neural progenitor cells in the subventricular zone by regulating $\mathrm{p} 53$ during homeostasis and following traumatic brain injury. Stem Cells 28(7):1231-1242

10. Kim H, Cooke MJ, Shoichet MS (2012) Creating permissive microenvironments for stem cell transplantation into the central nervous system. Trends Biotechnol 30(1):55-63

11. Mothe AJ, Tator $\mathrm{CH}$ (2012) Advances in stem cell therapy for spinal cord injury. J Clin Invest 122(11):3824-3834

12. Wang $X$ et al (2015) Macrophages in spinal cord injury: phenotypic and functional change from exposure to myelin debris. Glia 63(4):635-651

13. Kigerl KA et al (2009) Identification of two distinct macrophage subsets with divergent effects causing either neurotoxicity or regeneration in the injured mouse spinal cord. $\mathrm{J}$ Neurosci 29(43):13435-13444

14. Xu Q et al (2007) Hypoxia-induced astrocytes promote the migration of neural progenitor cells via vascular endothelial factor, stem cell factor, stromal-derived factor-1alpha and monocyte chemoattractant protein-1 upregulation in vitro. Clin Exp Pharmacol Physiol 34(7):624-631

15. van der Meulen AA et al (2009) The role of CXC chemokine ligand (CXCL)12-CXC chemokine receptor (CXCR)4 signalling in the migration of neural stem cells towards a brain tumour. Neuropathol Appl Neurobiol 35(6):579-591

16. Itoh T et al (2009) The relationship between SDF-1alpha/CXCR4 and neural stem cells appearing in damaged area after traumatic brain injury in rats. Neurol Res 31(1):90-102

17. Zhang K et al (2015) Polarized Macrophages Have Distinct Roles in the Differentiation and Migration of Embryonic Spinal-cord-derived Neural Stem Cells After Grafting to Injured Sites of Spinal Cord. Mol Ther 23(6):1077-1091

18. Wang $X$ et al (2012) MIF produced by bone marrow-derived macrophages contributes to teratoma progression after embryonic stem cell transplantation. Cancer Res 72(11):2867-2878

19. Vogel DY et al (2014) Macrophages migrate in an activation-dependent manner to chemokines involved in neuroinflammation. J Neuroinflammation 11:23 
20. McWhorter FY et al (2013) Modulation of macrophage phenotype by cell shape. Proc Natl Acad Sci U S A 110(43):17253-17258

21. Reynolds BA, Tetzlaff W, Weiss S (1992) A multipotent EGF-responsive striatal embryonic progenitor cell produces neurons and astrocytes. J Neurosci 12(11):4565-4574

22. Khan T et al (1999) Animal models of spinal cord contusion injuries. Lab Anim Sci 49(2):161-172

23. Kirik OV, Vlasov TD, Korzhevskii DE (2012) [Nestin and Musashi1 as the markers of neural stem cells in rat telencephalon following transitory focal ischemia]. Morfologiia, 142(4): p. 19-24

24. Andres RH et al (2011) The CCR2/CCL2 interaction mediates the transendothelial recruitment of intravascularly delivered neural stem cells to the ischemic brain. Stroke 42(10):2923-2931

25. Jarnagin $\mathrm{K}$ et al (1999) Identification of surface residues of the monocyte chemotactic protein 1 that affect signaling through the receptor CCR2. Biochemistry 38(49):16167-16177

26. Widera D et al (2004) MCP-1 induces migration of adult neural stem cells. Eur J Cell Biol 83(8):381387

27. Cerioni L, Palomba L, Cantoni O (2003) The Raf/MEK inhibitor PD98059 enhances ERK1/2 phosphorylation mediated by peroxynitrite via enforced mitochondrial formation of reactive oxygen species. FEBS Lett 547(1-3):92-96

28. Vogel $S$ et al (2010) Prolyl hydroxylase domain (PHD) 2 affects cell migration and F-actin formation via RhoA/rho-associated kinase-dependent cofilin phosphorylation. J Biol Chem 285(44):3375633763

29. D'Angeli S, Altamura MM (2007) Osmotin induces cold protection in olive trees by affecting programmed cell death and cytoskeleton organization. Planta 225(5):1147-1163

30. Liu XS et al (2007) Chemokine ligand 2 (CCL2) induces migration and differentiation of subventricular zone cells after stroke. J Neurosci Res 85(10):2120-2125

31. Zhou X, He X, Ren Y (2014) Function of microglia and macrophages in secondary damage after spinal cord injury. Neural Regen Res 9(20):1787-1795

32. Brown GC (2007) Mechanisms of inflammatory neurodegeneration: iNOS and NADPH oxidase. Biochem Soc Trans 35(Pt 5):1119-1121

33. Gordon S, Taylor PR (2005) Monocyte and macrophage heterogeneity. Nat Rev Immunol 5(12):953964

34. Gupta A, Pansari K (2003) Inflammation and Alzheimer's disease. Int J Clin Pract 57(1):36-39

35. Aarum J et al (2003) Migration and differentiation of neural precursor cells can be directed by microglia. Proc Natl Acad Sci U S A 100(26):15983-15988

36. Foresti ML et al (2009) Chemokine CCL2 and its receptor CCR2 are increased in the hippocampus following pilocarpine-induced status epilepticus. J Neuroinflammation 6:40

37. Collington SJ et al (2010) The role of the CCL2/CCR2 axis in mouse mast cell migration in vitro and in vivo. J Immunol 184(11):6114-6123 
38. Hodge DL et al (2012) MCP-1/CCR2 interactions direct migration of peripheral B and T lymphocytes to the thymus during acute infectious/inflammatory processes. Eur J Immunol 42(10):2644-2654

39. Yan C et al (2016) Human umbilical cord mesenchymal stem cells delivering sTRAlL home to lung cancer mediated by MCP-1/CCR2 axis and exhibit antitumor effects. Tumour Biol 37(6):8425-8435

40. Belema-Bedada $\mathrm{F}$ et al (2008) Efficient homing of multipotent adult mesenchymal stem cells depends on FROUNT-mediated clustering of CCR2. Cell Stem Cell 2(6):566-575

41. Chen $Y$ et al (2003) Overexpression of monocyte chemoattractant protein 1 in the brain exacerbates ischemic brain injury and is associated with recruitment of inflammatory cells. J Cereb Blood Flow Metab 23(6):748-755

42. Glabinski AR et al (1996) Chemokine monocyte chemoattractant protein-1 is expressed by astrocytes after mechanical injury to the brain. J Immunol 156(11):4363-4368

43. Bluthgen N, Legewie S (2008) Systems analysis of MAPK signal transduction. Essays Biochem 45:95-107

44. Yang Q et al (2014) 38 MAPK signal pathway involved in anti-inflammatory effect of Chaihu-ShuganSan and Shen-ling-bai-zhu-San on hepatocyte in non-alcoholic steatohepatitis rats. Afr J Tradit Complement Altern Med 11(1):213-221

45. LaRusch GA et al (2010) Factor XII stimulates ERK1/2 and Akt through uPAR, integrins, and the EGFR to initiate angiogenesis. Blood 115(24):5111-5120

46. Gong $X$ et al (2006) Stromal cell derived factor-1 acutely promotes neural progenitor cell proliferation in vitro by a mechanism involving the ERK1/2 and PI-3K signal pathways. Cell Biol Int 30(5):466471

47. Moors $\mathrm{M}$ et al (2007) ERK-dependent and -independent pathways trigger human neural progenitor cell migration. Toxicol Appl Pharmacol 221(1):57-67

\section{Figures}




\section{Fig. 1}

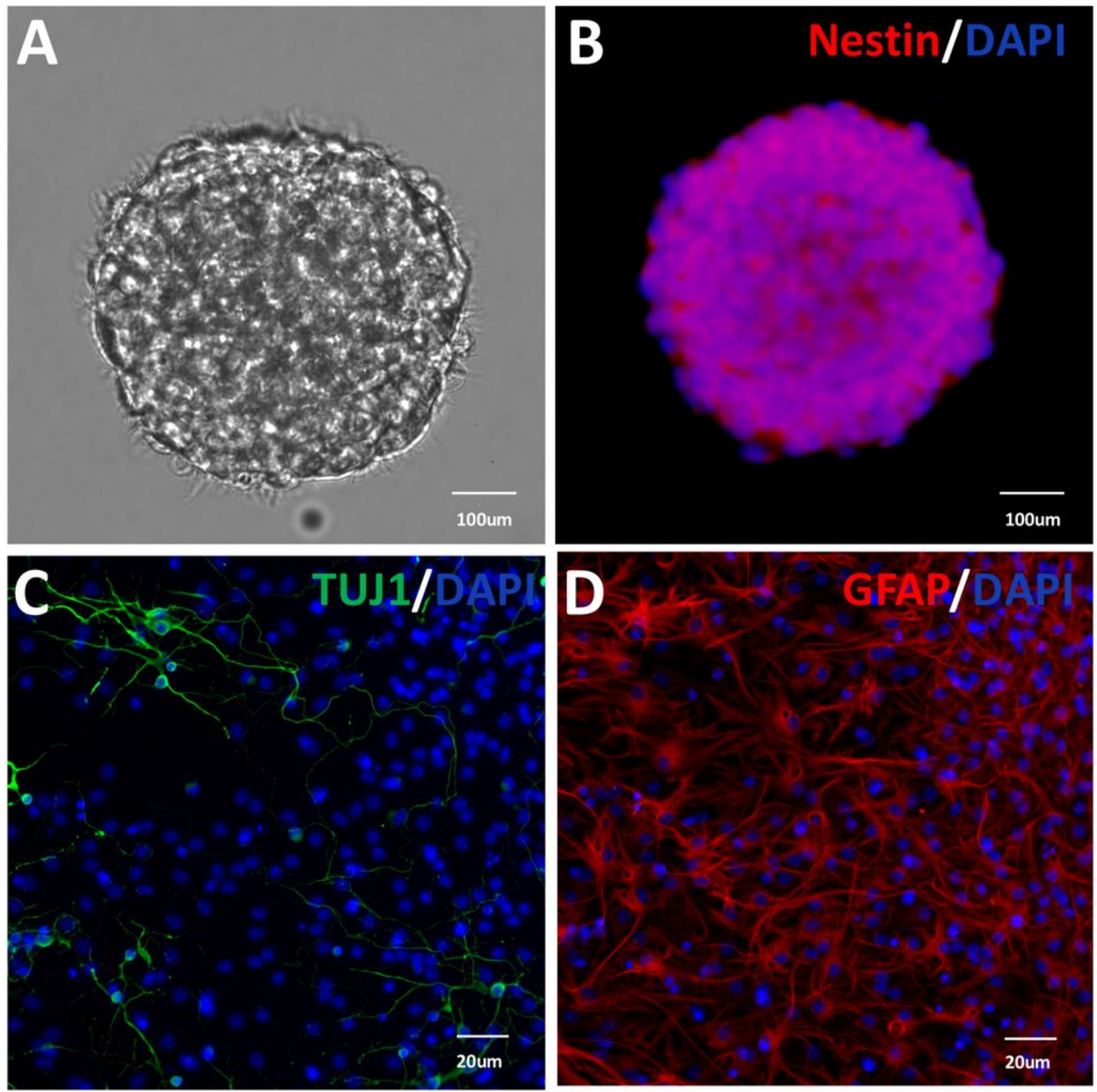

\section{Figure 1}

Identification of NSCs from embryonic mouse brain. After 7days cultured in serum free media including B27, EGF and bFGF, NSCs proliferated and formed neurosphere (A). Meanwhile, the neurosphere expressed nestin (Red, B), a marker of neural stem/progenitor cells. After 5 days differentiation in the media without the mitogens, the cells expressed the neuronal marker TUJ1 (Green, C), the astrocytic marker glial fibrillary acidic protein (GFAP) (Red, D). So, NSCs exhibited the capacity to differentiate into neurons, astrocytes and oligodendrocytes. Nuclei in C-D was stained with DAPI (Blue). 
Fig. 2

A

Lower well

Control

MO-CM

M1-CM

Up well

WT NSCS

WT NSCs

WT NSCS
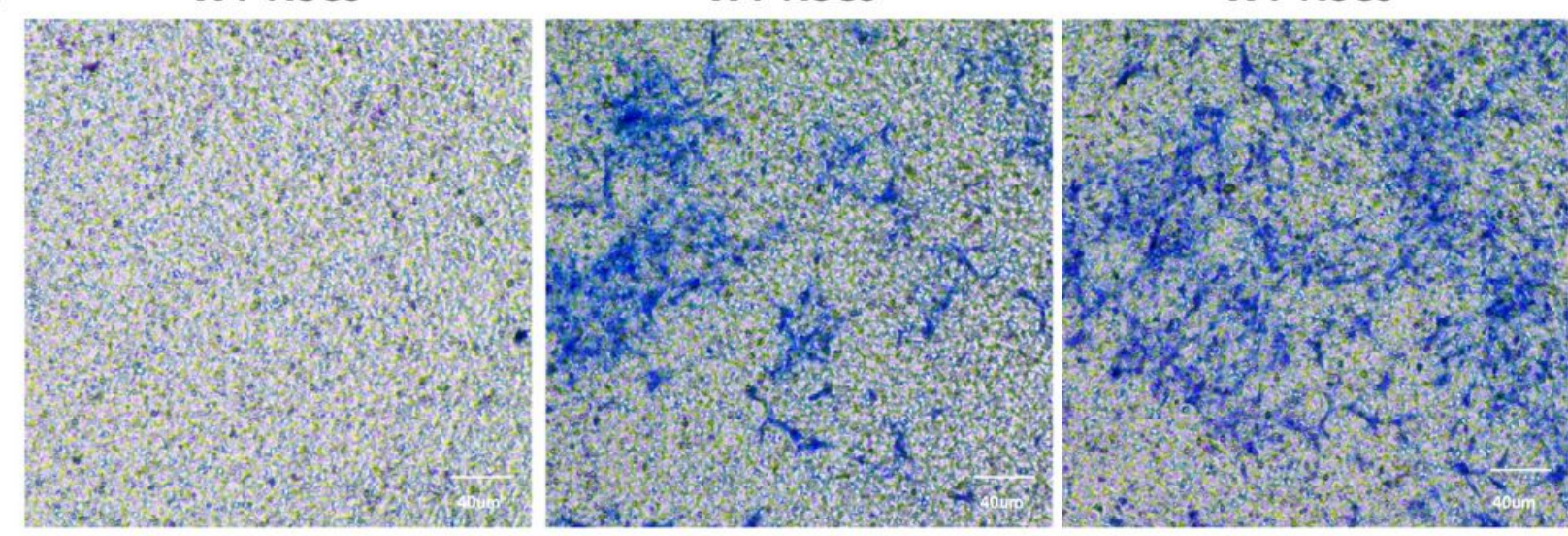

B

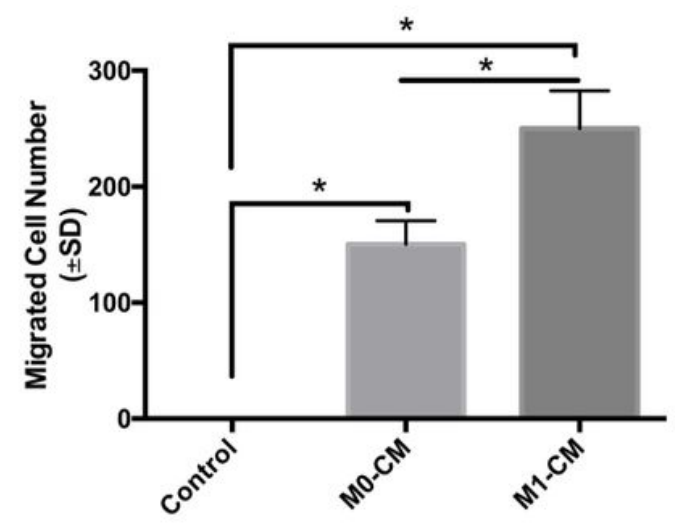

C

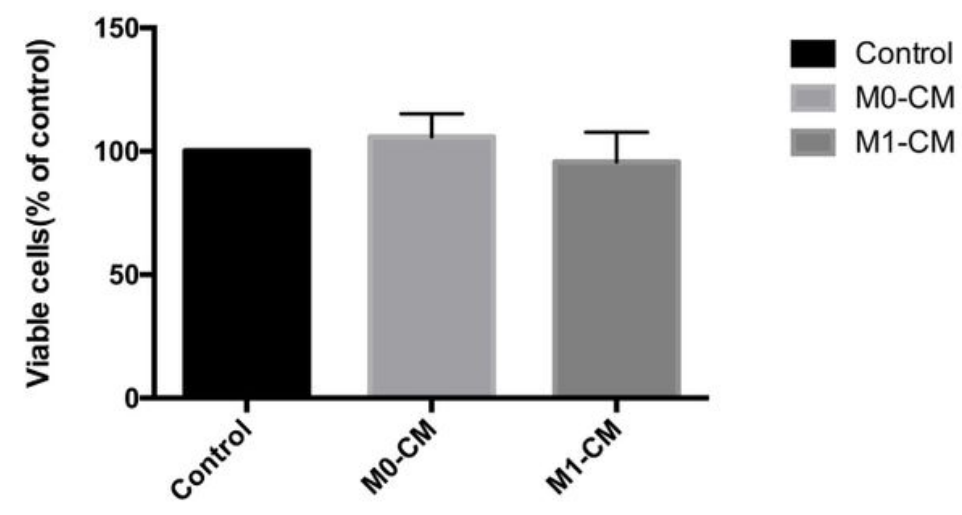

Figure 2

Conditioned medium from BMDMs promoted migration of mouse WT NSCs. A. Migration of NSCs upon exposure to $\mathrm{M} 1-\mathrm{CM}$ and $\mathrm{M} 0-\mathrm{CM}$ was detected using the transwell chamber with a pore size of $8 \mu \mathrm{m}$ (Neuro probe). Serum free DMEM containing 2\% B-27 supplement (without BMDMs) was used as experimental control (scale bar $=40 \mu \mathrm{m}$ ). B. Quantitative result of the migrated cell number of NSCs towards conditioned media from macrophage $(n=6, * P<0.05)$. C. The proliferation of NSCs was treated with M0-CM, M1-CM and control medium for $12 \mathrm{hMTT}$ assay and then detected using MTT assay. There were no significant effects of M1-CM and control medium on the proliferation of NSCs. Relative proliferation rates were presented as fold increase compared with control group ( $n=6, P>0.05)$. 


\section{Fig. 3}
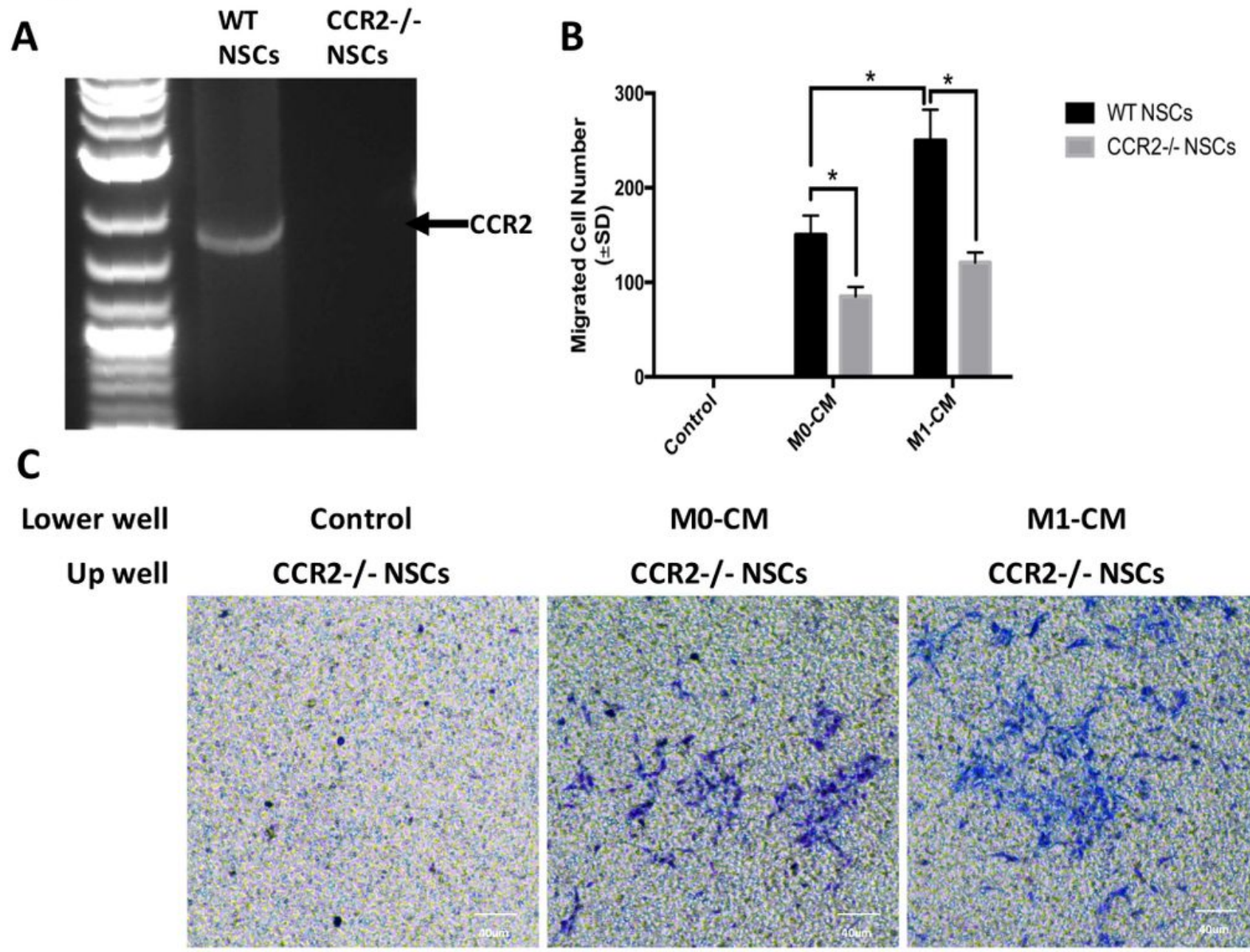

Lower well
Up well

Control

MO-CM

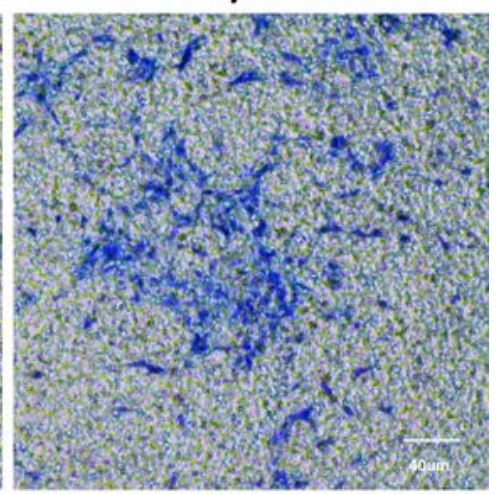

Control
WT NSCS

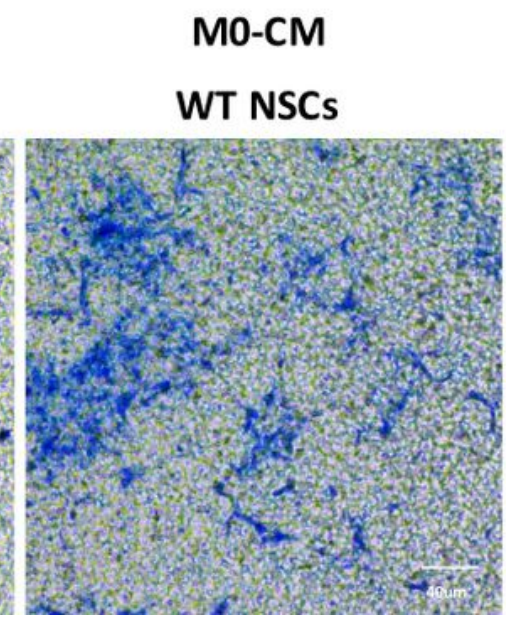

M1-CM
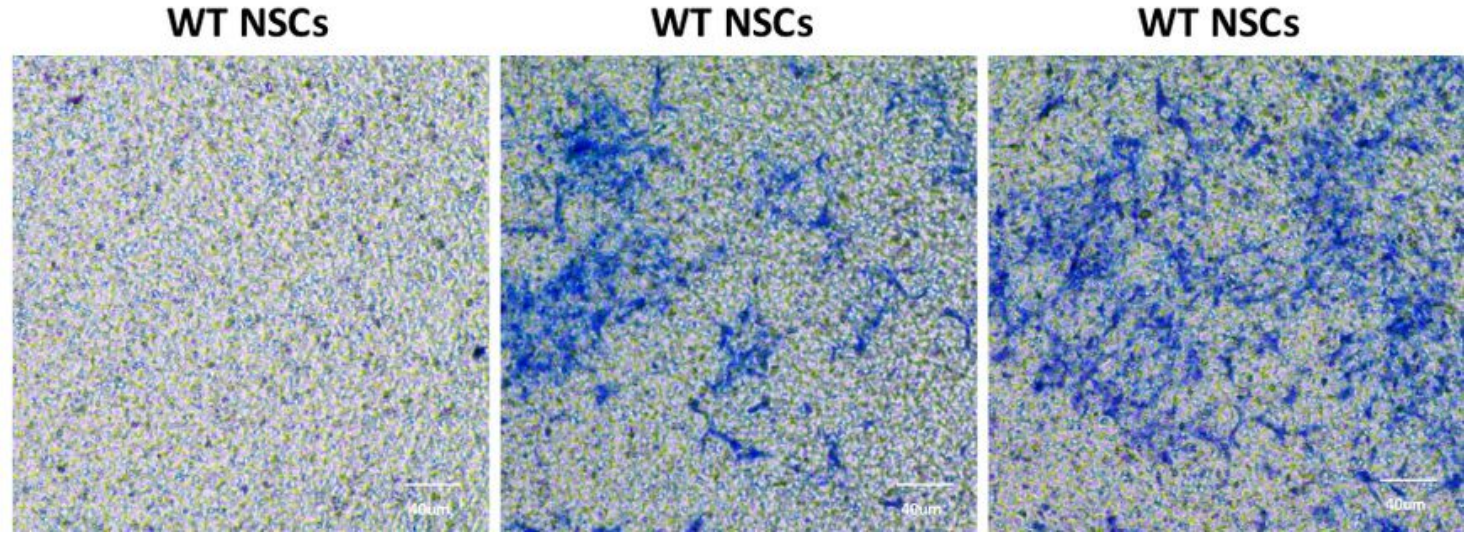

Figure 3

M1-CM induced migration of NSCs depending on CCR2. A. The expression of CCR2 in WT NSCs and CCR2-/- NSCs were confirmed by reverse transcription polymerase chain reaction. B. Quantitative result of the migrated cell number of WT NSCs and CCR2-/- NSCs towards M1-CM ( $\left.n=6,{ }^{*} P<0.05\right)$. C. CCR2-/- NSCs showed reduced migration toward M1-CM vs. WT NSCs. 


\section{Fig. 4}
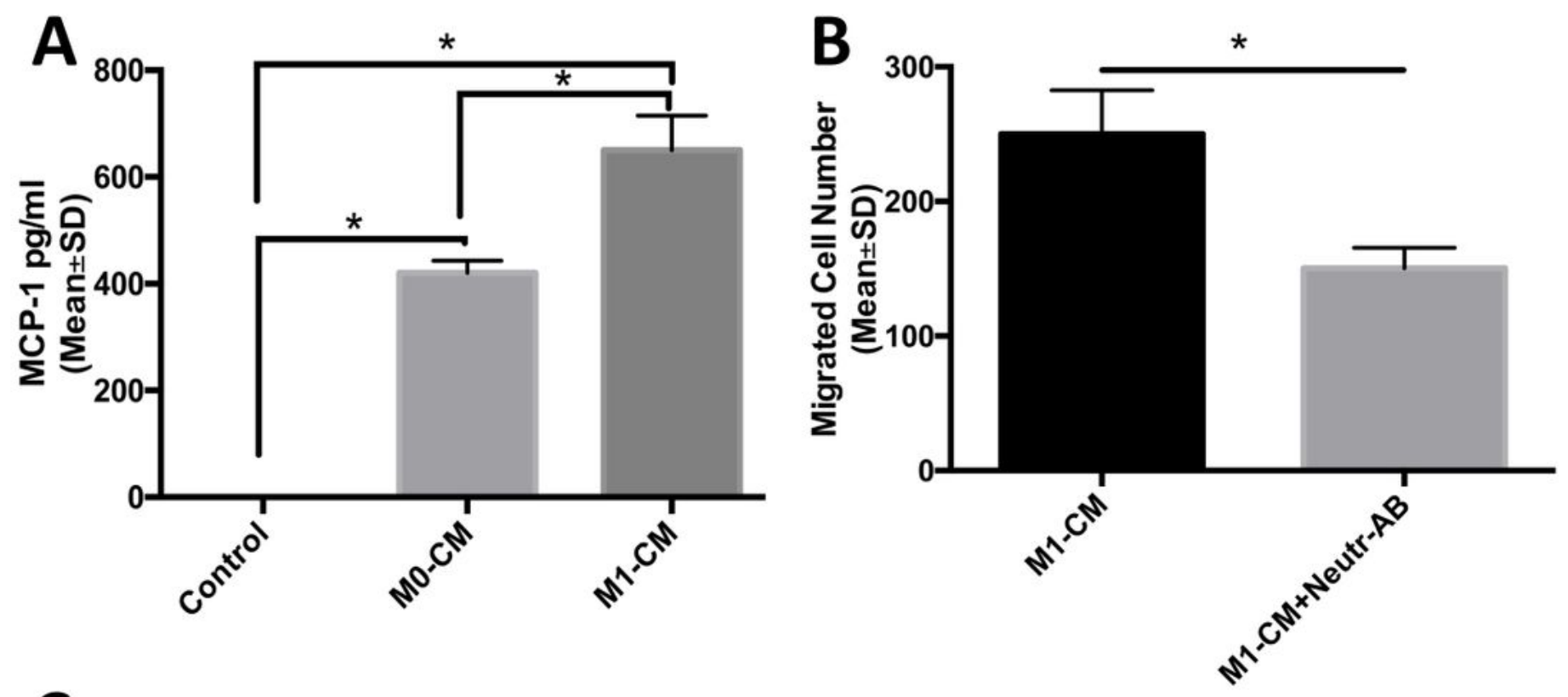

C

\section{Lower well}

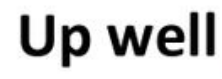

M1-CM WT NSCs

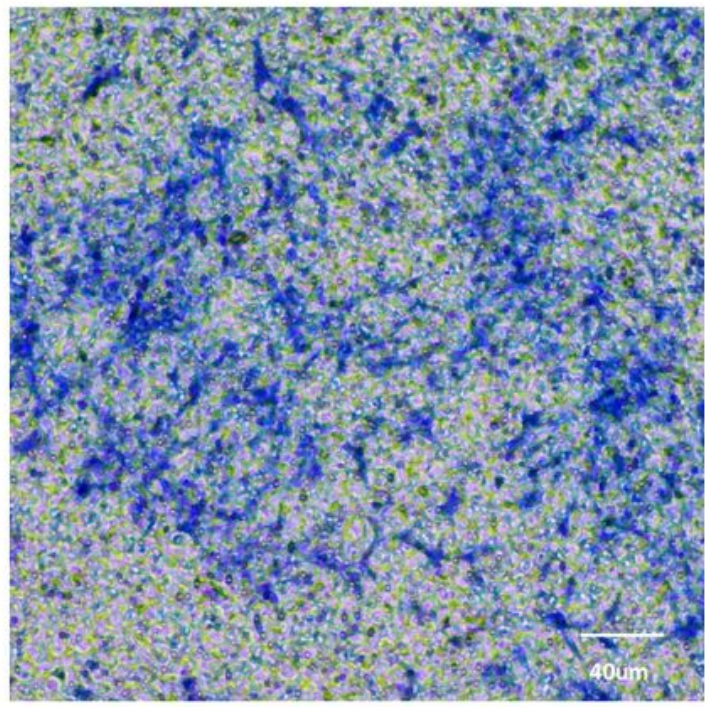

M1-CM+Neutr-AB WT NSCS

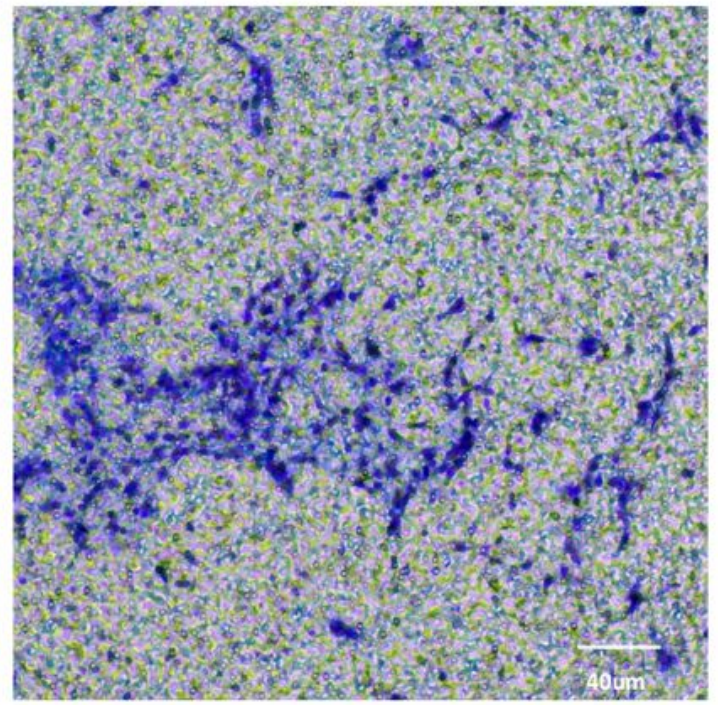

Figure 4

Role of MCP1 on the M1-CM-mediated NSCs migration. A. The production of MCP-1 by M1 macrophages was detected using ELISA $\left(n=6,{ }^{*}<0.05\right)$. B. Quantitative result of the inhibited migration of NSCs toward M1-CM by anti-MCP-1 antibody to neutralize MCP-1 in M1-CM. Data are mean \pm SD $\left(n=6,{ }^{*}<0.05\right)$. C. Antibody neutralization of MCP-1 abolished M1-CM-mediated NSCs migration. 


\section{Fig. 5}

\section{A}

Lower well

M1-CM

M1-CM

Up well

WT NSCs

WT NSCs+PD98059
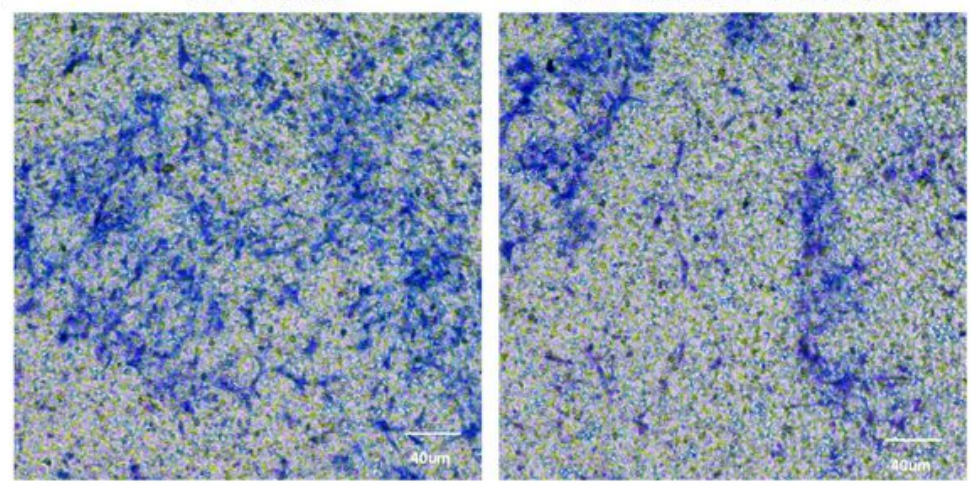

B

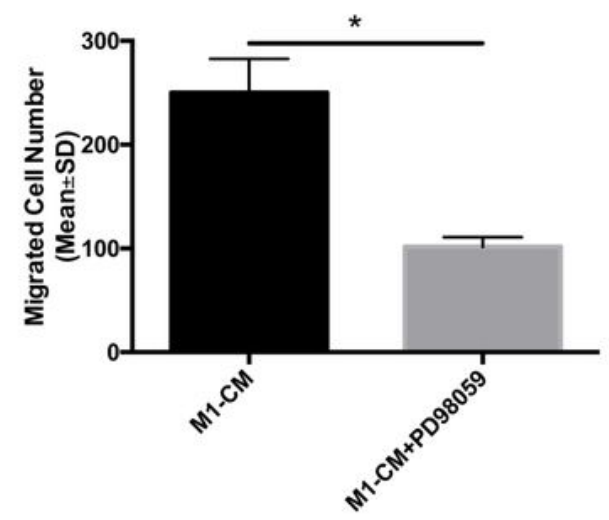

M1-CM

M1-CM+PD98059

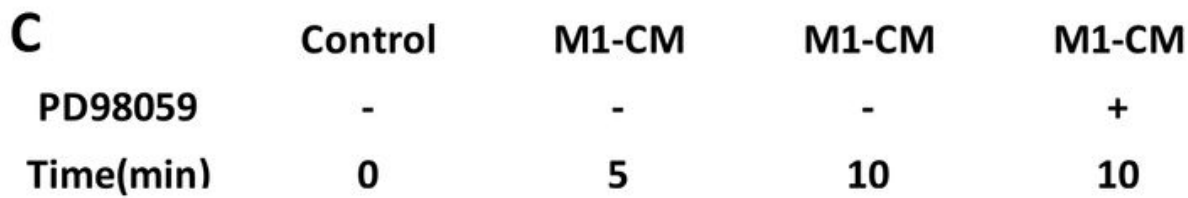

p-ERK1/2

ERK1/2

GAPDH

\section{Figure 5}

Inhibition of M1-CM-induced NSCs migration by PD98059. A. The migration of NSCs towards M1-CM can be inhibited by PD98059, an inhibitor of ERK pathway. B. Quantitative result of the inhibited migration of NSCs toward M1-CM by PD98059 $(n=6, * P<0.05)$. C. Western blot analysis of the upregulation of ERK activity by M1-CM. 


\section{Fig. 6}

\section{Control}

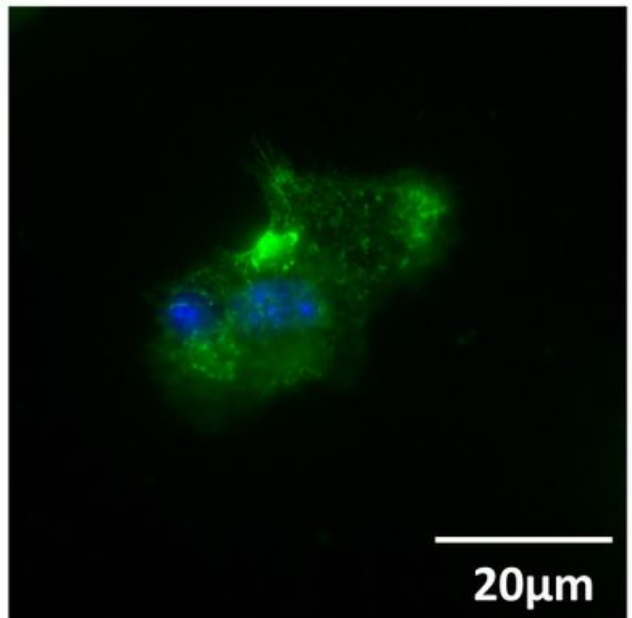

$\mathrm{MO}-\mathrm{CM}$

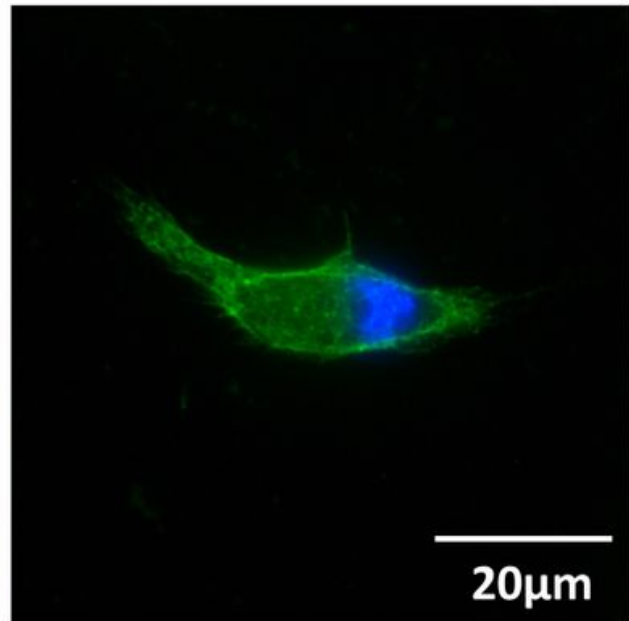

M1-CM

F-actin/

Figure 6

The expression and distribution of F-actin. Immunofluorescence analysis was performed using CF488conjugated phalloidin (F-actin, green). Nuclei were stained with DAPI (Blue). Scale bar $20 \mu \mathrm{m}$.

\section{Fig. 7}

\section{A WT NSCs}
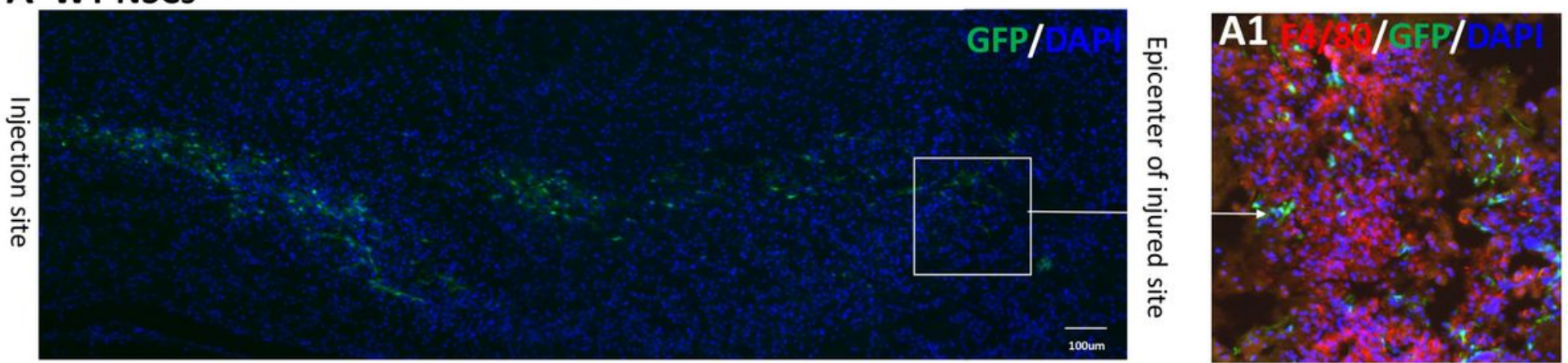

\section{B CCR2-/- NSCs}
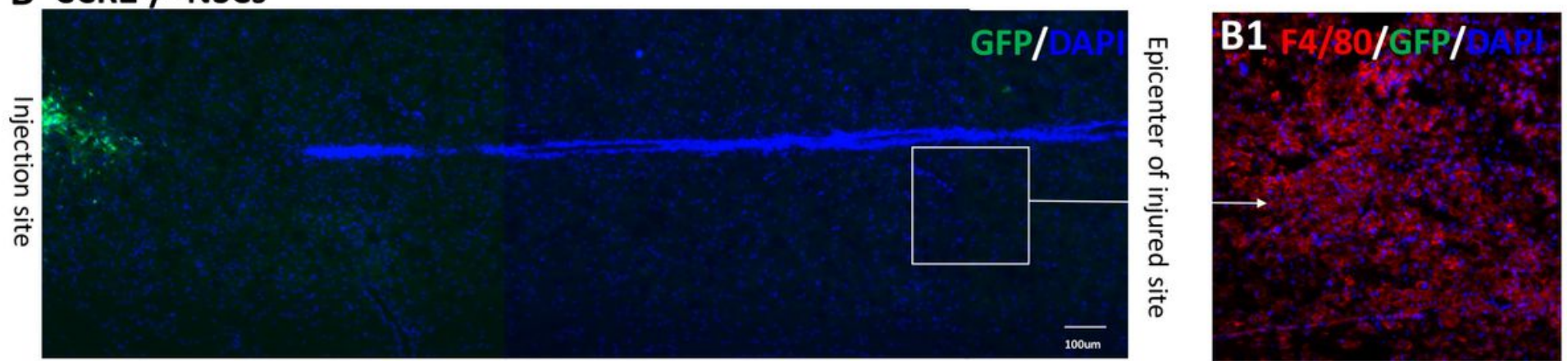

Figure 7 
Distribution of wild-type NSCs and CCR2-/- NSCs in the spinal cord 5days after Cells transplantation in WT C57BL/6 mice. A. Distribution of microinjected NSCs from WT mouse pre-labeled with CFSE in the spinal cord 5days after transplantation in WT mice, green: WT NSCs (scale bar $=100 \mu \mathrm{m}$ ). A1. Both WT NSCs (Green) and F4/80 positive (Red) cells were found in the epicenter of injury area. B. Distribution of microinjected NSCs from CCR2-/- mouse pre-labeled with CFSE in the spinal cord 5days post cells transplantation in WT mice, green: CCR2-/- NSCs (scale bar= $100 \mu \mathrm{m}$ ). B1. Only microglia/macrophages marker $F 4 / 80$ positive cells (Red) were observed in the epicenter of damaged site, without GFP positive cells. Nuclei was stained with DAPI (Blue).

\section{Fig. 8}
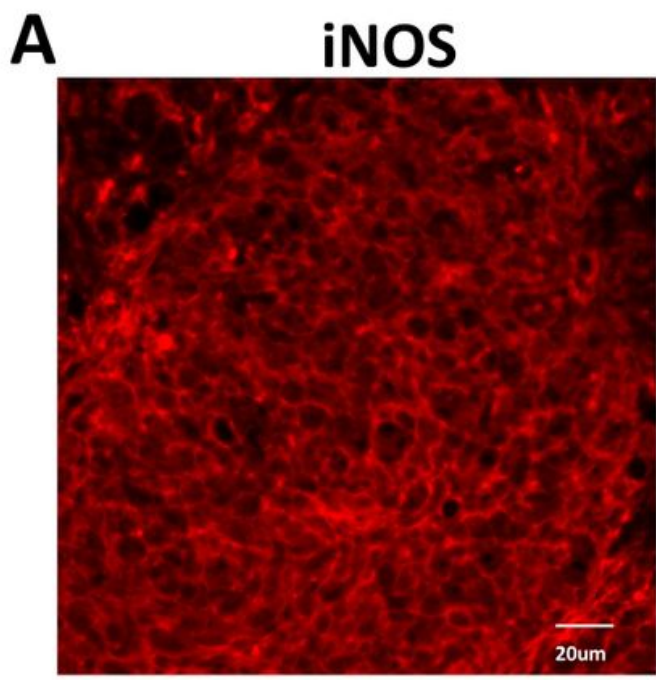

Mac-2

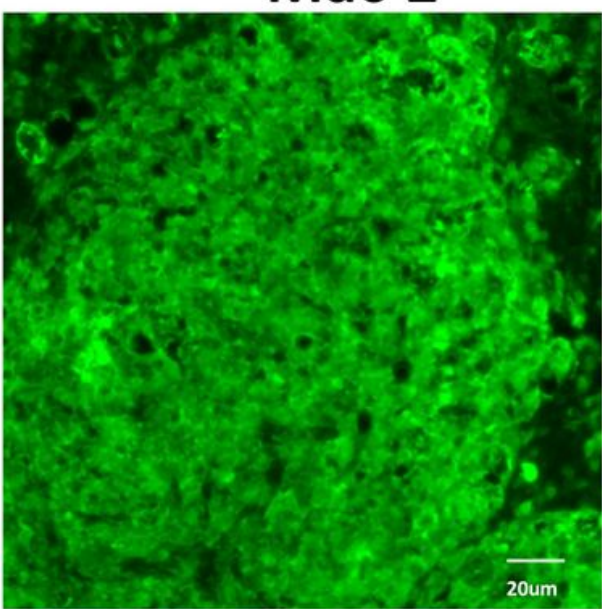

Merge

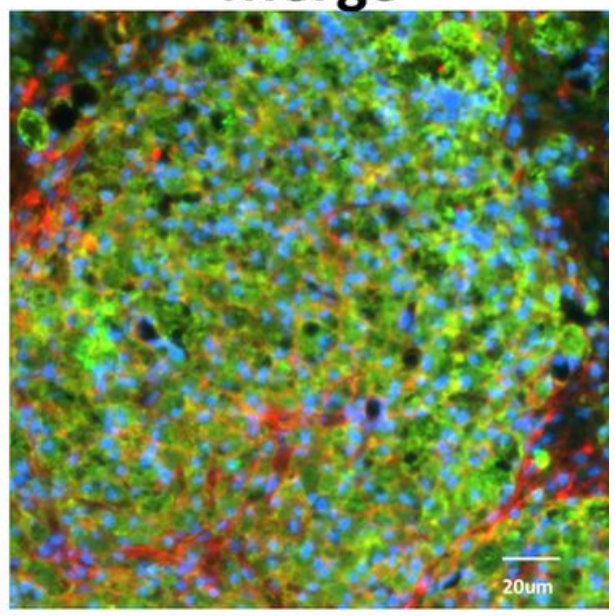

B

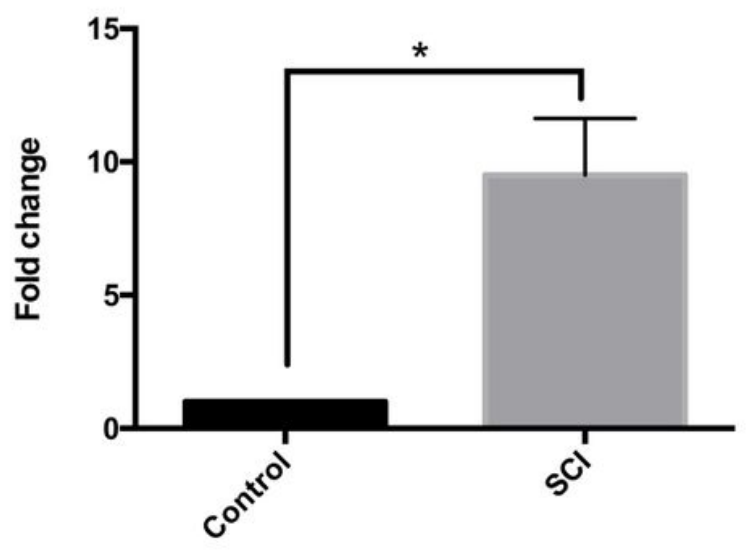

Control $\mathrm{SCl}$

\section{Figure 8}

M1 macrophages expressed and mRNA level of MCP-1 increased in spinal cord after SCl. 7days after SCl, the injured spinal cords were collected to detect the expression of INOS by using immunofluorescence staining (A) and mRNA level of MCP-1 using RT-PCR (B). A. Representative images of spinal cord sections with iNOS (Red) and Mac-2 (Green) double staining. Nuclei was stained with DAPI (Blue). B. MRNA level of MCP-1 was significantly increased in the $S C l$ group compared with sham group $(n=6, P<0.05)$. 
Bar $=20 \mu \mathrm{m}$, shown in (A and B). Results are displayed as Mean \pm Standard Error. ${ }^{*} P<0.05$. NSCs, Neural stem cells. 